\title{
Clinically Significant Unclassified Variants in BRCA1 and BRCA2 Genes among Korean Breast Cancer Patients
}

\author{
Kyong-Ah Yoon, $\mathrm{PhD}^{1,2}$ \\ Boyoung Park, MD, PhD \\ Byung II Lee, $\mathrm{PhD}^{4}$ \\ Moon Jung Yang, BS ${ }^{1}$ \\ Sun-Young Kong, MD, PhD 5,6,7 \\ Eun Sook Lee, MD, PhD $1,6,8$
}

${ }^{1}$ Center for Breast Cancer, Hospital, National Cancer Center, Goyang, ${ }^{2}$ College of Veterinary Medicine, Konkuk University, Seoul, ${ }^{3}$ Department of Cancer Control and Policy, Graduate School of Cancer Science and Policy, National Cancer Center, Goyang,

${ }^{4}$ Biomolecular Function Research Branch, National Cancer Center, Goyang,

${ }^{5}$ Genetic Counseling Clinic, Hospital,

National Cancer Center, Goyang,

${ }^{6}$ Department of System Cancer Science,

Graduate School of Cancer Science and Policy, National Cancer Center, Goyang,

${ }^{7}$ Translational Epidemiology Branch, National Cancer Center, Goyang, ${ }^{8}$ Precision Medicine Branch, Research Institute, National Cancer Center, Goyang, Korea

Correspondence: Sun-Young Kong, MD, PhD Genetic Counseling Clinic, Hospital, Department of System Cancer Science, Graduate School of Cancer Science and Policy, Translational Epidemiology Branch,

Research Institute, National Cancer Center, 323 Ilsan-ro, Ilsandong-gu, Goyang 10408, Korea Tel: 82-31-920-1735

Fax: 82-31-920-1738

E-mail: ksy@ncc.re.kr

\section{Co-correspondence: Eun Sook Lee, MD, PhD}

Center for Breast Cancer, Hospital,

Department of System Cancer Science,

Graduate School of Cancer Science and Policy,

Precision Medicine Branch, Research Institute,

National Cancer Center, 323 Ilsan-ro,

Ilsandong-gu, Goyang 10408, Korea

Tel: 82-31-920-1503

Fax: 82-31-920-1511

E-mail: eslee@ncc.re.kr

Received July 5, 2016

Accepted September 13, 2016

Published Online September 13, 2016

\section{Purpose}

Unclassified variants (UVs) of BRCA1 and BRCA2 genes are not defined as pathogenic for breast cancer, and their clinical significance currently remains undefined. Therefore, this study was conducted to identify potentially pathogenic UVs by comparing their prevalence between breast cancer patients and controls.

\section{Materials and Methods}

A total of 328 breast cancer patients underwent BRCA1/2 genetic screening at the National Cancer Center of Korea. Genetic variants of BRCA genes that were categorized as unclassified according to the Breast Cancer Information Core database were selected based on allelic frequency, after which candidate variants were genotyped in 421 healthy controls. We also examined family members of the study participants. Finally, the effects of amino acid substitutions on protein structure and function were predicted in silico.

\section{Results}

Genetic tests revealed $33 \mathrm{UVs}$ in BRCA1 and 47 in BRCA2. Among 15 candidates genotyped in healthy controls, c.5339T $>\mathrm{C}$ in BRCA1 and c.6029T $>\mathrm{G}$, c.7522G $>\mathrm{A}$ in BRCA2 were not detected. Moreover, the c.5339T $>C$ variant in the BRCA1 gene was detected in four patients with a family history of breast cancer. This nonsynonymous variant (Leu1780Pro) in the BRCA1 C-terminal domain was predicted to have an effect on BRCA1 protein structure/function.

\section{Conclusion}

This study showed that comparison of genotype frequency between cases and controls could help identify UVs of BRCA genes that are potentially pathogenic. Moreover, our findings suggest that c.5339T>C in BRCA1 might be a pathogenic variant for patients and their families.

Key words

Familial breast cancer, BRCA1, BRCA2, Unclassified variants 


\section{Introduction}

Breast cancer is the second-most common cancer among women in Korea, with an estimated incidence of 65.7 per 100,000 women per year [1]. Moreover, the incidence of breast cancer in Korea has been increasing annually, with relatively younger-aged women increasingly being affected.

Germline mutations of the BRCA1 and BRCA2 genes that encode truncated proteins are associated with a significantly increased risk of cancer in carriers [2-4]. The Korean Hereditary Breast Cancer (KOHBRA) study reported that $15.7 \%$ of patients with breast cancer who were tested for genetic mutation carried pathogenic mutations in BRCA genes. Additionally, breast cancer patients with a family history of breast or ovarian cancers showed a prevalence of $B R C A$ mutations as high as $22.3 \%[5,6]$.

Mutation screening for BRCA genes has become a widely applied genetic test for cancer predisposition. Currently, the clinical significance of $B R C A 1 / 2$ sequence variations can be interpreted according to several databases of genetic mutation, including the Breast Cancer Information Core (BIC; http:// research.nhgri.nih.gov/bic/) and ClinVar (http:// www.ncbi.nlm.nih.gov/clinvar/) [7-9]. However, a large portion of genetic variants of $B R C A 1$ and $B R C A 2$ genes are nontruncating, such as missense or potential splice site changes. Nevertheless, the contribution of these variants to cancer risk currently remains undefined. These unclassified variants (UVs) of $B R C A$ genes have become a clinical issue for carriers because of their unknown clinical significance $[10,11]$. Some UVs that are found in highly conserved domains or splice sites have been predicted to be deleterious by in silico analyses. Moreover, many UVs are classified as neutral polymorphisms, and some are considered potentially deleterious. The effects of variants on biological function are difficult to assign based on functional assays of $B R C A$ genes. To define the clinical significance of UVs, researchers have suggested various approaches and algorithms to determine whether UVs are deleterious or neutral for the biological function of proteins encoded by BRCA genes [12,13]. Many models based on statistical methods that combine clinical features or predicted gene function with informatics tools, such as Polymorphism Phenotyping (PolyPhen, http://genetics. bwh.harvard.edu/pph/) or Sorting Intolerant from Tolerant (SIFT, http://blocks.fhcrc.org/sift/SIFT.html), have been suggested [14,15].

In this study, we investigated the prevalence of UVs in $B R C A$ genes in a Korean population. To address the clinical significance of unclassified $B R C A$ gene variants, we collected $B R C A$ gene sequencing data from 328 breast cancer patients. Additionally, six selected UVs of the BRCA1 gene and nine of the $B R C A 2$ gene were genotyped in 421 controls. We also examined the family history of variant carriers and tested $B R C A$ genes of family members. This is the first report comparing the frequency of BRCA UVs in Korean breast cancer patients and healthy controls.

\section{Materials and Methods}

\section{Study population}

Patients with histologically confirmed breast cancer were enrolled in this study from the genetic counseling clinic and underwent BRCA1/2 mutation testing between April 2008 and June 2015 at the National Cancer Center in Korea. A total of 328 patients who underwent genetic testing for $B R C A 1$ and $B R C A 2$ genes voluntarily participated in this study and agreed to provide the results of genetic testing. As control group, 421 healthy controls were recruited from individuals who visited the National Cancer Center as part of a cancerscreening program. All individuals who participated in this study signed an informed consent form that was approved by the Institutional Review Board of National Cancer Center Korea (IRB No. NCCNCS 13717).

\section{Sequencing and genotyping of variants}

Genomic DNA was extracted from the peripheral blood of participants using a QIAamp DNA Blood Mini kit (Qiagen, Valencia, CA) or a Chemagic DNA Blood 200 Kit (Chemagen, Baesweiler, Germany) according to the manufacturers' instructions. Genetic testing of $B R C A 1$ and $B R C A 2$ genes was conducted by the Green Cross Company (Yongin, Korea) using a direct sequencing method. Briefly, amplified products were sequenced on an ABI 3500xl Analyzer (Applied Biosystems, Foster City, CA) using Bigdye Terminator v3.1 Cycle Sequencing Kits, and sequences were analyzed using the Sequencer v5.0 software. All genetic variants in BRCA1 and $B R C A 2$ genes were categorized as pathogenic, unclassified, or polymorphic according to the BIC database. All mutations are described according to HUGO-approved systematic nomenclature (http://www.hgvs.org/mutnomen/). GenBank accession numbers NM_007294.3 for BRCA1 and NM_000059.3 for BRCA2 were used as reference sequences.

Large genomic rearrangements of $B R C A 1 / 2$ genes were also tested using a multiplex ligation-dependent probe amplification (MLPA) assay for patients without pathogenic mutations of the $B R C A$ genes.

Candidate variants were selected for further genotyping in healthy controls based on the frequency of variants of $B R C A 1 / 2$ genes among cases. Variants were identified by 
TaqMan probe genotyping (Applied Biosystems) using a QuantStudio 7 Flex real-time PCR system. The reproducibility of genotyping results was confirmed by genotyping $10 \%$ of the samples in duplicate.

\section{In silico analysis of UVs}

The effects of amino acid substitutions on protein structure and function were predicted using PolyPhen [16] and SIFT [17]. The tolerance score from SIFT and damaging score from PolyPhen-2 were used to predict the potential effects of UVs on the function of proteins encoded by BRCA genes. The structure of variant proteins was predicted using SWISSMODEL (http://swissmodel.expasy.org/) [18]. The BRCA1 C-terminal (BRCT) domain structure of BRCA1 (PDB entry: $4 \mathrm{U} 4 \mathrm{~A}$ ) was used as a template for modeling.

\section{Results}

\section{Patient characteristics}

Table 1 shows the demographic features of breast cancer patients and controls. All patients were female and were diagnosed with histologically confirmed breast cancer, with the exception of four patients who were diagnosed with ovarian cancer. Patients in our study population were predominantly stage I, with only 11 patients categorized as stage IV. Additionally, more than $76 \%$ of patients had a family history of breast or ovarian cancer, including 11 with a family history of both breast and ovarian cancer.

All patients underwent genetic testing of BRCA1 and $B R C A 2$ genes by the direct sequencing method. As shown in Tables 1 and 2, a total of 47 patients $(14.3 \%)$ harbored 35 different deleterious mutations (frameshift or nonsense) of $B R C A$ genes. To examine large genomic rearrangement of the $B R C A$ genes, we performed MLPA assays in 196 patients. Only four patients showed deletions of a large genomic region.

\section{UVs of $B R C A 1 / 2$ genes}

Sequencing results showed that a total of 181 patients (55.2\%) harbored UVs of BRCA genes. Among these, 33 kinds of UVs in the BRCA1 gene were detected in 127 patients, while 47 UVs in the BRCA2 gene were identified from 113 patients. Although these variants were already classified as having uncertain clinical importance, such a high frequency of variants among the population could weaken the significance of the association with cancer. Therefore, we excluded
Table 1. Demographic characteristics of breast cancer patients tested for $B R C A 1 / 2$ genes

\begin{tabular}{|cc}
\hline Characteristic & No. (\%) \\
\hline Female & 328 \\
\hline Current age, median (range, yr) & $44(25-76)$ \\
\hline Age at cancer diagnosis, median (range, yr) & $43(25-73)$ \\
\hline Classification of cancer type & \\
\hline Invasive ductal carcinoma & $236(72.0)$ \\
\hline Ductal carcinoma in situ & $36(11.0)$ \\
\hline Invasive lobular carcinoma & $18(5.5)$ \\
\hline Lobular carcinoma in situ & $4(1.2)$ \\
\hline Others & $34(10.4)$ \\
\hline Stage of breast cancer & \\
\hline Stage 0 & $40(12.2)$ \\
\hline Stage I & $124(37.8)$ \\
\hline Stage II & $102(31.1)$ \\
\hline Stage III & $47(14.3)$ \\
\hline Stage IV & $11(3.4)$ \\
\hline Unknown & $4(1.2)$ \\
\hline Family history & \\
\hline Breast cancer & $216(65.9)$ \\
\hline Ovarian cancer & $25(7.6)$ \\
\hline Breast and ovarian cancer & $11(3.4)$ \\
\hline Without family history & $76(23.2)$ \\
\hline Pathogenic mutation carrier & \\
\hline BRCA1 pathogenic variant & $20(6.1)$ \\
\hline BRCA2 pathogenic variant & $27(8.2)$ \\
\hline MLPA tested patients & $113(34.5)$ \\
\hline BRCA1 rearrangement carrier & $3(0.9)$ \\
\hline Patients with BRCA2 unclassified variant & 0 \\
\hline
\end{tabular}

Control ( $\mathrm{n}=421)$ : current age, 45 (27-71) years. MLPA, multiplex ligation-dependent probe amplification assay.

14 UVs that were present at a frequency of more than $2 \%$ among the East-Asian population in the 1000 Genomes Phase 3 database (http: // www.1000genomes.org/ data). Seven UVs in BRCA1, including rs1799949, rs799912, rs16940, rs799916, rs1060915, rs3092994, and rs8176140, showed a minor allele frequency (MAF) of $37 \%$ in the East-Asian population. Furthermore, rs1801406, rs9534262, and rs4942486 in the BRCA2 gene showed reduced significance because their MAF was than $25 \%$. Ultimately, $19 \mathrm{UV}$ s that were detected in at least two patients among our cases were selected for further analysis; however, four UVs could not be genotyped owing to difficulties in probe design. Finally, six UVs in BRCA1 and 
Table 2. Genetic alterations in BRCA1 and $B R C A 2$ genes detected in 328 Korean breast cancer patients

\begin{tabular}{lcc} 
Genetic alteration & BRCA1 & BRCA2 \\
Pathogenic variants & 17 & 18 \\
Frameshift & 10 & 8 \\
Nonsense & 7 & 10 \\
Unclassified variants & 33 & 47 \\
1000 Genomes Phase 3 MAFs & 26 & 40 \\
in EAS (<0.02) & & \\
MAF in 328 breast cancer patients $(>0.005)$ & 7 & 12 \\
Genotyping in controls & 6 & 9 \\
\hline
\end{tabular}

MAF, minor allele frequency; EAS, East-Asian population.

nine in $B R C A 2$ were further genotyped in 421 age-matched female controls. Among these, c.5339T $>C$ in BRCA1 and c. $6029 \mathrm{~T}>\mathrm{G}$, c.7522G $>\mathrm{A}$ in BRCA2 were not detected in healthy controls (Table 3). The c.5339T $>C$ were detected in four patients and c.6029T>G, c.7522G $>$ A were detected in three patients with breast cancer. All three variants caused a substitution of amino acid sequence and c.5339T $>C$ (Leu1780Pro) in BRCA1, and c.7522G $>A$ (Gly2508Ser) in $B R C A 2$ were predicted as damaging variants. In contrast, c. $4883 \mathrm{~T}>\mathrm{C}$ and c. $2566 \mathrm{~T}>\mathrm{C}$ in $B R C A 1$ and c.2350A $>\mathrm{G}$ and c. $8187 \mathrm{G}>\mathrm{T}$ in $B R C A 2$ showed a genotype frequency greater than $2 \%$ in the control group.

\section{Potential risk of c.5339T $>C$ variant in the BRCA1 gene}

We examined the potential risk of three UVs that were not detected in 421 healthy controls. Fortunately, we were able to recruit family members of the proband harboring the c.5339T $>C$ variant in the BRCA1 gene. As shown in the pedigree in Fig. 1A, two breast cancer patients in this family and the proband were also diagnosed with ovarian cancer 2 years after being diagnosed with breast cancer. The father of the proband also carried the same UV, and his sister died of breast cancer at the age of 46 . Another patient who harbored the same variant was diagnosed with breast cancer at the age of 33, as shown in Fig. 1B. Her mother suffered from ovarian cancer and could not participate in this study. The c.5339T $>C$ variant results in an amino acid change from leucine to proline at position 1780 . The predicted structure shows that the mutation site is in the middle of a helix in the BRCT domain of BRCA1, forming a hydrophobic patch with its surrounding residues (Fig. 1C). The BRCT domain is known to recognize and bind phosphorylated pSXXF motifs of FAM175A/ Abraxas to recruit BRCA1 to regions of DNA damage [19-21].

\section{Discussion}

Interpreting UVs in $B R C A 1$ and $B R C A 2$ genes has become a particularly important issue for genetic counseling of cancer patients because of the clinical importance of germline mutations in BRCA genes. Here, we sought to define potentially pathogenic variants by comparing the prevalence of $B R C A$ UVs in 421 healthy controls and 328 breast cancer patients in a Korean population by genotyping. Among the 80 UVs that were found in our patients, 15 were identified in controls while three were detected only in patients with breast cancer, not in controls. Some of these latter variants were predicted to be "probably damaging" based on a high score in PolyPhen-2, and were classified as "intolerant" variants by the SIFT tool. Additionally, the nonsynonymous variant c.5339T>C, which causes an amino acid substitution of proline for leucine (Leu1780Pro) in the BRCT domain, was detected in the BRCA1 gene of four patients with breast cancer. The BRCT domain in the C-terminal, which is known to be essential for BRCA1 to function as a tumor suppressor [19], contributes to binding to target proteins with specificity for phosphorylated pSer-X-X-Phe motifs [20,21]. The substitution of proline for leucine may weaken the hydrophobic patch structure of the BRCT domain, potentially influencing the protein-protein interactions needed for the proper function of BRCA1.

The average age at diagnosis of four patients harboring the c. $5339 \mathrm{~T}>\mathrm{C}$ variant was 34 , and the youngest patient was diagnosed at the age of 25 . One breast cancer patient harboring the same variant was subsequently diagnosed with ovarian cancer following breast cancer, and the mother of one patient suffered from ovarian cancer. Based on these findings, it is plausible to suggest c.5339T $>\mathrm{C}$ as a potentially pathogenic variant.

Interestingly, one candidate UV in the $B R C A 2$ gene, c. $7522 \mathrm{G}>\mathrm{A}$, has been reported as a risk factor for breast cancer in a case-control study. This variant is a nonsynonymous single-nucleotide polymorphism known as rs80359878 that causes an amino acid substitution (Gly2508Ser) in BRCA2. Zhang et al. [22] showed that this missense variant was associated with a 16.5-fold increase in the risk of breast cancer among Chinese women, with an allele frequency of this variant of 0.0023 in cases and 0.0001 in controls.

To define rare variants with potential pathogenicity, we compared the frequency of UVs of BRCA genes among healthy controls with that in breast cancer patients. Our results suggest the potentially deleterious variants, c.5339T $>C$ (Leu1780Pro) in BRCA1 and c.6029T $>$ G (Val2010Gly), c.7522G $>$ A (Gly2508Ser) in BRCA2, which were detected only in cases. This strategy could be strengthened using a large number of cases-controls to select signifi- 


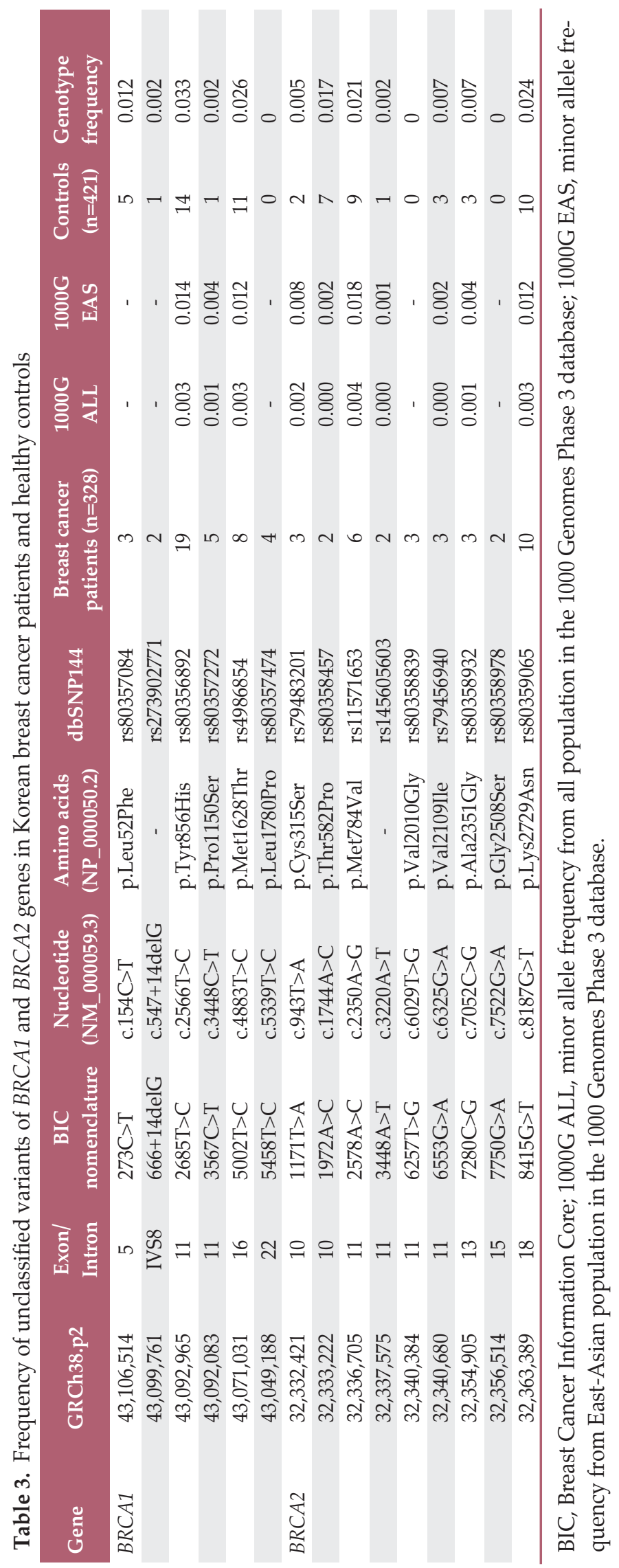



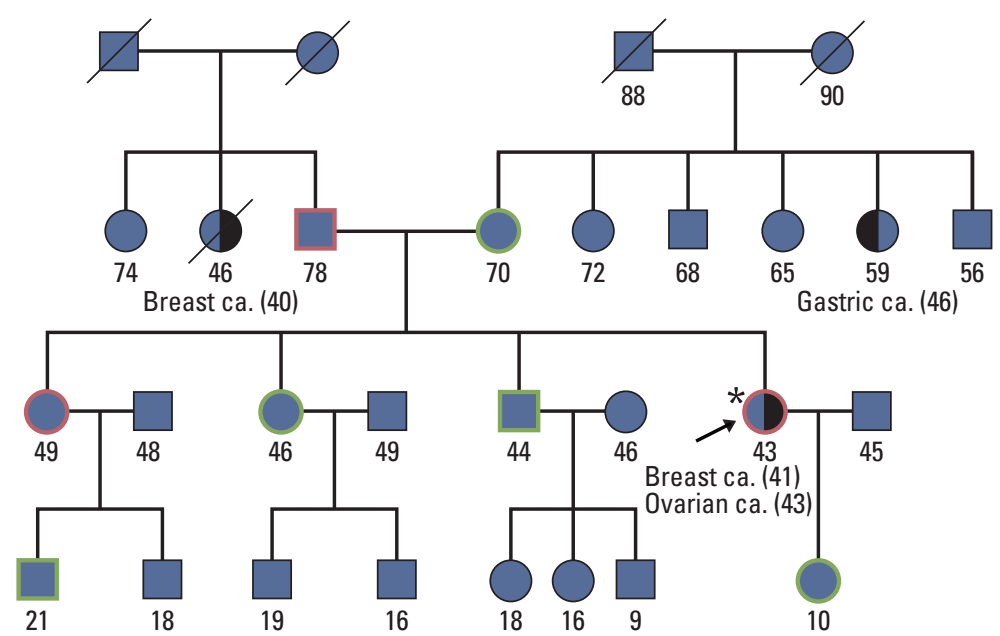

B

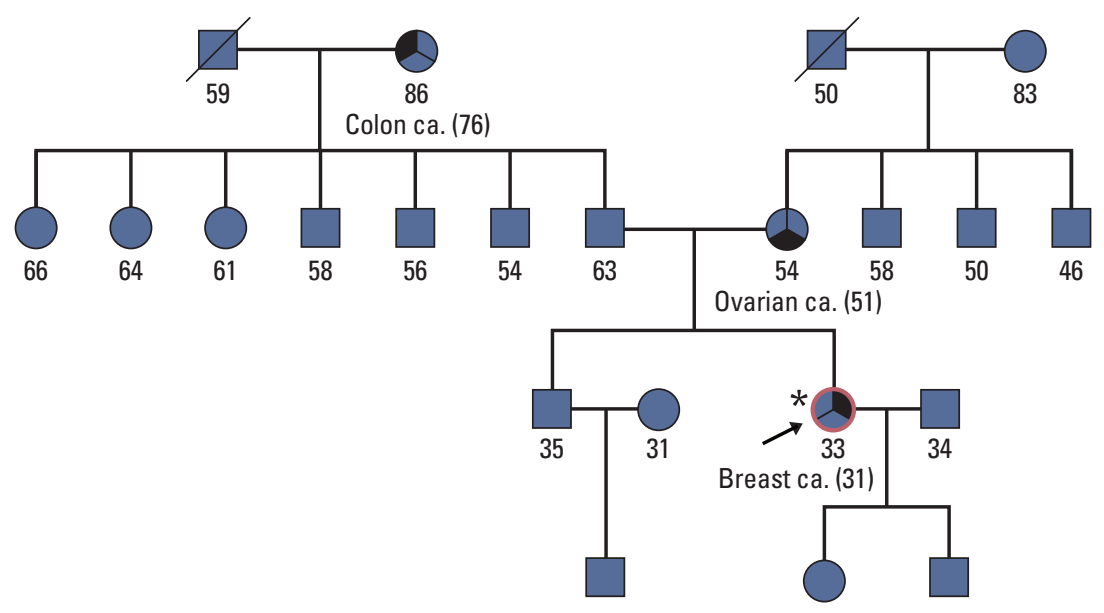

Fig. 1. Unclassified variant c.5339T $>C$ in $B R C A 1$. The candidate UV, c.5339T $>C$, was tested in breast cancer patients and family members (A, B). Red in each pedigree indicates a carrier of the variant genotype, while green indicates family members without the variant. The proband of each family is indicated by a black arrow. (Continued to the next page)

cant variants among previously UVs. Biological experiments should be performed to validate the effects of the variants. pathogenicity by affecting the function of the BRCT domain of BRCA1. The information provided herein will be useful for individuals carrying these variants, who should be carefully monitored for potential cancer risk.

\section{Conclusion}

In conclusion, the c.5339T $>C$ variant in BRCA1 that was detected in four patients may be involved in breast cancer 


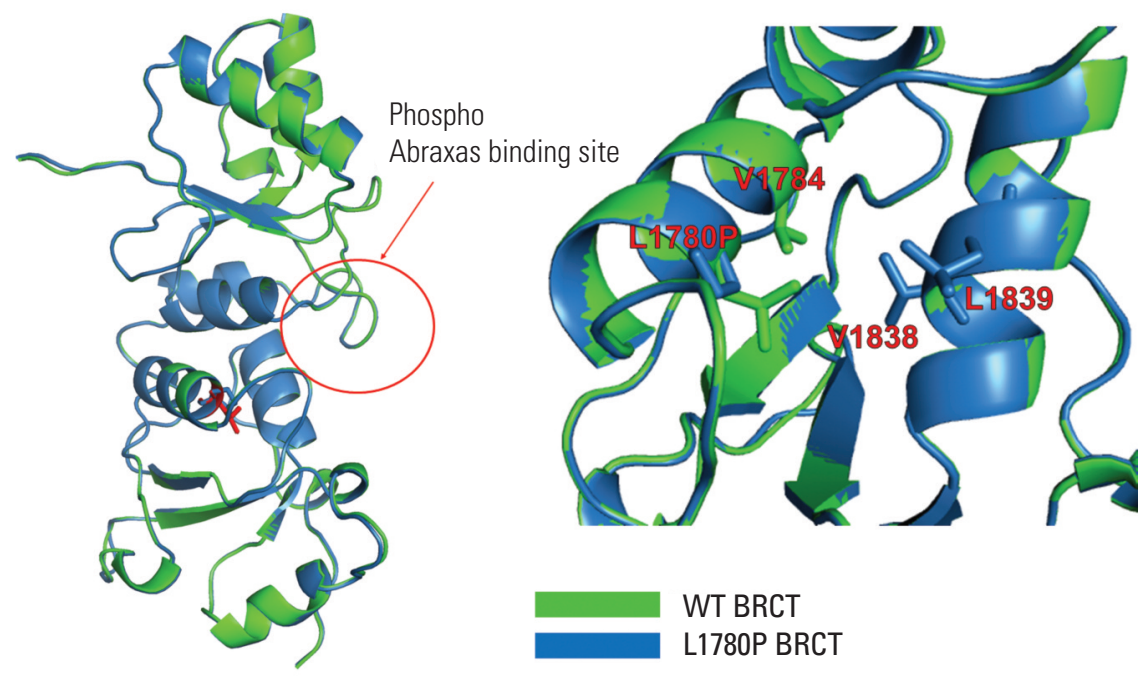

C

Fig. 1. (Continued from the previous page) (C) Predicted structure of BRCA1 variant (Leu1780Pro) in the BRCA1 C-terminal (BRCT) domain. Left, overall structure of the BRCT domain of BRCA1; right, detailed view of the region surrounding the variant. Hydrophobic residues around Leu1780 are shown and labeled in red.

\section{Conflicts of Interest}

Conflict of interest relevant to this article was not reported.

\section{Acknowledgments}

We thank the many individuals who participated in this study. We also thank Ms. Eun Ji Choi for her technical support. This work was supported by a grant from the National Cancer Center, Korea (1410691).

\section{References}

1. Jung KW, Won YJ, Kong HJ, Oh CM, Cho H, Lee DH, et al. Cancer statistics in Korea: incidence, mortality, survival, and prevalence in 2012. Cancer Res Treat. 2015;47:127-41.

2. Futreal PA, Liu Q, Shattuck-Eidens D, Cochran C, Harshman $\mathrm{K}$, Tavtigian $\mathrm{S}$, et al. BRCA1 mutations in primary breast and ovarian carcinomas. Science. 1994;266:120-2.

3. Miki Y, Swensen J, Shattuck-Eidens D, Futreal PA, Harshman $\mathrm{K}$, Tavtigian S, et al. A strong candidate for the breast and ovarian cancer susceptibility gene BRCA1. Science. 1994;266: 66-71.

4. Wooster R, Bignell G, Lancaster J, Swift S, Seal S, Mangion J, et al. Identification of the breast cancer susceptibility gene BRCA2. Nature. 1995;378:789-92.

5. Son BH, Ahn SH, Kim SW, Kang E, Park SK, Lee MH, et al. Prevalence of BRCA1 and BRCA2 mutations in non-familial breast cancer patients with high risks in Korea: the Korean
Hereditary Breast Cancer (KOHBRA) Study. Breast Cancer Res Treat. 2012;133:1143-52.

6. Kang E, Seong MW, Park SK, Lee JW, Lee J, Kim LS, et al. The prevalence and spectrum of BRCA1 and BRCA2 mutations in Korean population: recent update of the Korean Hereditary Breast Cancer (KOHBRA) study. Breast Cancer Res Treat. 2015;151:157-68.

7. Szabo C, Masiello A, Ryan JF, Brody LC. The breast cancer information core: database design, structure, and scope. Hum Mutat. 2000;16:123-31.

8. Landrum MJ, Lee JM, Riley GR, Jang W, Rubinstein WS, Church DM, et al. ClinVar: public archive of relationships among sequence variation and human phenotype. Nucleic Acids Res. 2014;42:D980-5.

9. Harrison SM, Riggs ER, Maglott DR, Lee JM, Azzariti DR, Niehaus A, et al. Using ClinVar as a resource to support vari- 
ant interpretation. Curr Protoc Hum Genet. 2016;89:8.16. 1-8.16.23.

10. Easton DF, Deffenbaugh AM, Pruss D, Frye C, Wenstrup RJ, Allen-Brady K, et al. A systematic genetic assessment of 1,433 sequence variants of unknown clinical significance in the BRCA1 and BRCA2 breast cancer-predisposition genes. Am J Hum Genet. 2007;81:873-83.

11. Calo V, Bruno L, La Paglia L, Perez M, Margarese N, Di Gaudio $F$, et al. The clinical significance of unknown sequence variants in BRCA genes. Cancers (Basel). 2010;2:1644-60.

12. Spearman AD, Sweet K, Zhou XP, McLennan J, Couch FJ, Toland AE. Clinically applicable models to characterize BRCA1 and BRCA2 variants of uncertain significance. J Clin Oncol. 2008;26:5393-400.

13. Gomez Garcia EB, Oosterwijk JC, Timmermans M, van Asperen CJ, Hogervorst FB, Hoogerbrugge N, et al. A method to assess the clinical significance of unclassified variants in the BRCA1 and BRCA2 genes based on cancer family history. Breast Cancer Res. 2009;11:R8.

14. Adzhubei IA, Schmidt S, Peshkin L, Ramensky VE, Gerasimova A, Bork $\mathrm{P}$, et al. A method and server for predicting damaging missense mutations. Nat Methods. 2010;7:248-9.

15. Flanagan SE, Patch AM, Ellard S. Using SIFT and PolyPhen to predict loss-of-function and gain-of-function mutations. Genet
Test Mol Biomarkers. 2010;14:533-7.

16. Adzhubei I, Jordan DM, Sunyaev SR. Predicting functional effect of human missense mutations using PolyPhen-2. Curr Protoc Hum Genet. 2013;Chapter 7:Unit7.20.

17. Sim NL, Kumar P, Hu J, Henikoff S, Schneider G, Ng PC. SIFT web server: predicting effects of amino acid substitutions on proteins. Nucleic Acids Res. 2012;40:W452-7.

18. Biasini M, Bienert S, Waterhouse A, Arnold K, Studer G, Schmidt T, et al. SWISS-MODEL: modelling protein tertiary and quaternary structure using evolutionary information. Nucleic Acids Res. 2014;42:W252-8.

19. Glover JN. Insights into the molecular basis of human hereditary breast cancer from studies of the BRCA1 BRCT domain. Fam Cancer. 2006;5:89-93.

20. Campbell SJ, Edwards RA, Glover JN. Comparison of the structures and peptide binding specificities of the BRCT domains of MDC1 and BRCA1. Structure. 2010;18:167-76.

21. Coquelle N, Green R, Glover JN. Impact of BRCA1 BRCT domain missense substitutions on phosphopeptide recognition. Biochemistry. 2011;50:4579-89.

22. Zhang Y, Long J, Lu W, Shu XO, Cai Q, Zheng Y, et al. Rare coding variants and breast cancer risk: evaluation of susceptibility Loci identified in genome-wide association studies. Cancer Epidemiol Biomarkers Prev. 2014;23:622-8. 